\section{Glaucoma treatment adherence at a United Kingdom general practice}

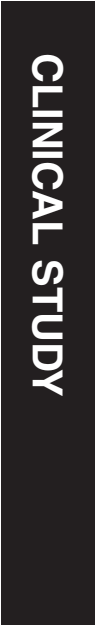

${ }^{1}$ Department of Ophthalmology, Stoke Mandeville Hospital, Aylesbury,

Buckinghamshire, UK

${ }^{2}$ Bassett Road Surgery, Leighton Buzzard, Bedfordshire, UK

Correspondence: A Shaikh, Department of Ophthalmology, Stoke Mandeville Hospital, Mandeville Road, Aylesbury, Buckinghamshire, HP21 8AL, UK

Tel: $+44(0) 1296316465$;

Fax: +44 (0)1296315789

E-mail: Asifa.Shaikh@

buckshealthcare.nhs.uk

Received: 16 August 2015 Accepted in revised form: 31 March 2016

Published online:

3 June 2016

\begin{abstract}
Purpose To estimate patient adherence with glaucoma therapy and identify factors associated with adherence using computerised patient prescribing records.

Methods Identification of patients diagnosed with glaucoma, ocular hypertension or suspect glaucoma and/or prescribed topical glaucoma medications registered at a United Kingdom general practice with 13422 patients. Adherence was defined as the average difference in the actual number of prescriptions collected annually compared to twelve prescriptions required annually (one bottle per month) over the duration of treatment.
\end{abstract}

Results Overall, 278 patients were identified of which $139(50 \%)$ were male. The average age was 72 years (range: $22-100$ ). A total of 206 patients $(74 \%)$ were prescribed glaucoma treatment. Adherence varied significantly between age groups with younger patients demonstrating poorest adherence $(P=0.0347)$. There was no statistical difference when comparing medication class, diagnosis, co-morbidities, or the number of drops being taken.

Conclusions Glaucoma treatment adherence improves with increasing age. Older patients require more prescriptions and may be experiencing drop wastage. Younger patients should be targeted with educational interventions to improve their understanding of glaucoma, and older patients for drop technique review. General practices are well placed to provide such interventions.

Eye (2016) 30, 1118-1122; doi:10.1038/eye.2016.103; published online 3 June 2016

\section{Introduction}

Glaucoma is one of the leading causes of blindness globally. ${ }^{1}$ In 2010, the World Health Organisation (WHO) estimated that 39 million
AP Tse ${ }^{1}$, M Shah' ${ }^{1}$, N Jamall ${ }^{2}$ and A Shaikh ${ }^{1}$

people in the world were blind, of which $8 \%$ were due to glaucoma. Approximately $2 \%$ of people over the age of 40 suffer with chronic open angle glaucoma (COAG) with up to $10 \%$ of white Europeans over 75 years being affected. Currently, this equates to $\sim 480000$ people being affected with COAG in England accounting for over 1 million hospital eye service visits annually, a figure likely to rise with an aging population. In the United Kingdom (UK) 10\% of blindness registrations are as a result of glaucoma. $^{2}$

Treatment of ocular hypertension (OHT) and glaucoma prevents visual loss and centres around reduction in intraocular pressure (IOP) either medically and/or surgically. ${ }^{3}$ Medical treatment of glaucoma using eye drops is the most common form of initial treatment and requires good adherence. ${ }^{4}$ Adherence is defined as the degree to which a patient uses medication as prescribed and persistence as the time interval between initiating and discontinuing a medication. Glaucoma is a chronic asymptomatic condition, usually requiring lifelong treatment and follow-up; adherence and persistence with glaucoma medications has been shown to be poor. ${ }^{5,6}$ This can be due to a number of factors, which include potential or perceived side effects of medications, ${ }^{7}$ lack of information about glaucoma, patient beliefs, ${ }^{8}$ treatment regime, ${ }^{9}$ and socio-demographic characteristics. ${ }^{10,11}$ Non-adherence to glaucoma medications can lead to avoidable visual loss. ${ }^{12}$

With an aging population placing an increased burden on hospital eye services, distribution of resources and management of conditions in primary care is becoming increasingly important. Ophthalmologists can have difficulty in accurately determining patient adherence with glaucoma treatment during short clinic visits. General practitioners are uniquely positioned to identify poorly compliant patients as they provide repeat prescriptions for 
medications. Electronic medication monitoring via issued repeat prescriptions offers an objective method of measuring adherence without reliance on patient self-reporting. ${ }^{13}$ GPs are well placed to identify those who have poor glaucoma treatment adherence, providing an opportunity for any issues such as difficulty in administering eye drops or inability to request repeat prescriptions due to time constraints in younger patients, to be addressed in the primary care setting. This study looks at glaucoma treatment adherence at a UK general practice (GP).

\section{Material and methods}

This study was conducted at a GP surgery in the UK with 13422 registered patients. Prospective approval from the clinical governance team of the local Clinical Commissioning Group was gained and the study was registered as an audit within the GP surgery. The General Practice was chosen as it falls within the secondary care catchment area and is representative of others within the local area. Primary, secondary and tertiary healthcare services are provided free at the point of delivery (through the National Health Service (NHS)) in the UK, but access is generally coordinated in the primary care setting by the patient's General Practitioner, particularly for secondary care services. GPs are therefore notified of planned or emergency healthcare utilisation outside of primary care (eg, hospital admissions, emergency department or out-patient attendances) and together with the primary care consultations, information is held on medical diagnoses, general health information, and prescriptions.

This information is recorded in the electronic medical record (EMR) of each patient and entries are made either by the GP or by an allied professional in the general practice using READ codes that are based on the International Classification of Diseases version 10 (ICD-10).

Computerised patient records identified subjects using glaucoma-related READ codes recorded in the patient's healthcare record at any point, while they were registered at the practice. In addition, to identify any patients who may not have had a glaucoma-related diagnosis READ code entered, all patients who were prescribed any ocular hypotensive eye drops were also included. The British National Formulary was used to populate a list for ocular hypotensive drugs by both generic and brand names. ${ }^{14}$ Data collection was performed over two consecutive days. All patients who were registered at the GP practice and were previously prescribed any ocular hypotensive eye drops or had a glaucoma-related READ code within their EMR were identified. Relevant details from their entire patient record were subsequently reviewed.
Adherence was defined as the average difference in the actual number of prescriptions collected annually compared to 12 prescriptions required annually over the duration of treatment. This was calculated as anerage per patient per drug.

We also assessed patient risk factors for poor adherence available in the GP held patient record, which included age, diagnosis (glaucoma, suspect glaucoma or OHT), co-morbidities, the number of different glaucoma eye drops prescribed, and class of prescribed topical ocular hypotensive medications. Owing to the small sample size, we grouped patients into age classes for the purposes of analysis of adherence. There is no significance in the choice of age classes selected.

All statistical analysis was performed using GraphPad InStat version 3.05 for Windows 95 (GraphPad Software, San Diego, CA, USA). Data was analysed using the Kruskal-Wallis test with post-test and the Mann-Whitney test as appropriate. Non-parametric statistical tests were used as data did not pass normality testing. A $P$-value $<0.05$ was considered statistically significant.

\section{Results}

A total of 278 patients were identified having either a READ code related to glaucoma or as being previously prescribed topical IOP lowering eye drops. A total of 139 patients $(50 \%)$ were male and the average age was 72 years (range: $22-100)$. Of these, 206 patients (74\%) were prescribed glaucoma treatment and were analysed further (see Table 1).

Adherence varied significantly between age groups ( $H=10.364,4 \mathrm{df}, P=0.0347$ ) (Figure 1). Patients up to the age of 59 years collected on average 2.3 fewer prescriptions per year and those aged 90-99 years collected on average 1.3 more prescriptions per year than the 12 required. Overall, patients collected on average 12.4 prescriptions per year.

The overall calculated adherence rate of all patients based on 12 prescriptions being required per year was $100 \%$. However, this was lower in the younger age group (20-59) at 62\% and higher in the older age group (90-99) at $111 \%$. Overall, over half $(51.6 \%)$ of patients demonstrated poor adherence (defined as <100\%).

There was no statistically significant difference in adherence between different medication classes ( $H=7.268,3 \mathrm{df}, P=0.0638$ ) (Figure 2). Equally, there was no statistical difference when comparing diagnoses ( $H=1.974,2 \mathrm{df}, P=0.3726$, glaucoma, suspect glaucoma, or OHT), co-morbidities $(H=0.4108,2 \mathrm{df}, P=0.8449)$, or the number of drops being taken $(H=0.6490,2 \mathrm{df}$, $P=0.7229)$. 
Table 1 Patient information by age range

\begin{tabular}{|c|c|c|c|c|c|c|}
\hline \multirow[t]{2}{*}{ Age range } & \multirow[t]{2}{*}{ Number } & \multicolumn{4}{|c|}{ Medication class $^{\mathrm{b}}$} & \multirow[t]{2}{*}{ Adherence $(\%)^{c}$} \\
\hline & & PGA $(\%)$ & CAI $(\%)$ & $A A(\%)$ & $B B(\%)$ & \\
\hline $20-59$ & 19 & 36.8 & 23.7 & 10.5 & 30 & 62 \\
\hline $60-69$ & 53 & 44.3 & 15 & 5.3 & 35.4 & 100 \\
\hline 70-79 & 70 & 54.7 & 14.9 & 4.9 & 25.5 & 100 \\
\hline 80-89 & 52 & 52.6 & 18.8 & 6.8 & 21.8 & 103 \\
\hline 90-99 & 12 & 52.8 & 22.2 & 11.1 & 13.9 & 111 \\
\hline
\end{tabular}

a Number of patients within that age range.

bercentage of patients within that age range who have been prescribed that medication class (PGA, prostaglandin analogues; CAI, carbonic anhydrase inhibitors; AA, alpha-adrenergic agonists; BB, beta blockers).

${ }^{c}$ Overall adherence rate for that age range calculated on the assumption that 12 prescriptions are required per year.

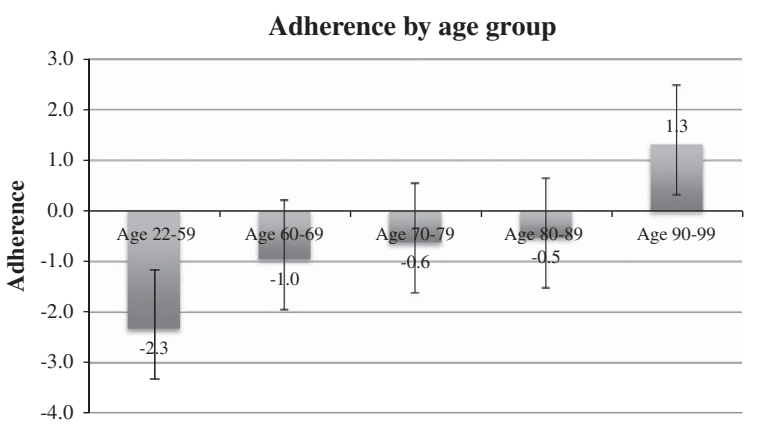

Figure 1 Adherence by age group. Adherence calculated as the average difference in number of prescriptions collected per year over the duration of treatment (assuming one prescription required per month). Error bars represent standard error of the mean.

\section{Discussion}

Using routinely collected data from a UK GP, this study showed that overall, adherence with treatment for glaucoma is poor. This is in keeping with other studies from both within and outside of the UK. 8,15,16

Glaucoma treatment adherence varied significantly with age. A systematic review by Olthoff et $a l^{17}$ did not identify a relationship between age and non-compliance. However, they acknowledged that this could just represent a lack of reporting of this relationship. They commented that it was unclear in some studies that did not report a relationship whether there was none, or that it was just not reported. Younger age groups were found to be less compliant. This could be due to a more active family lifestyle causing forgetfulness to use eye drops or working patterns leading to longer periods of time away from home. There could be a lack of patient education on the importance of taking their treatment, with younger patients considering glaucoma to be an 'older persons' disease. In addition, time constraints may not allow patients to request repeat prescriptions during working

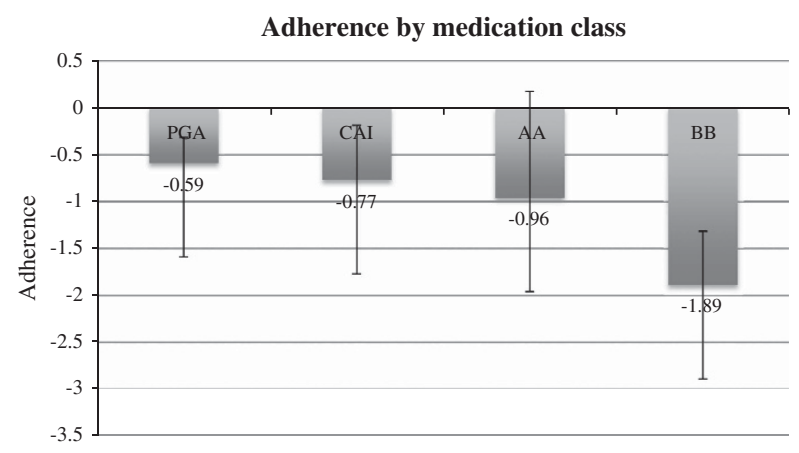

Figure 2 Adherence by medication class (PGA, prostaglandin analogues; CAI, carbonic anhydrase inhibitors; AA, alphaadrenergic agonists; BB, beta blockers). Adherence calculated as the average difference in number of prescriptions collected per year over the duration of treatment (assuming one prescription required per month). Error bars represent standard error of the mean.

hours. Interventions directed at helping patients understand the importance of medication adherence in preventing sight loss may be of benefit.

Patients from older age groups, particularly those over 90 years, seemed to collect more prescriptions than should be required. Difficulty in instilling the medication leading to drop wastage in this group as a result of physical challenges ${ }^{4,18,19}$ may account for the increased number of prescriptions, as patients would run out of medications before the expected refill date; we did not find a statistically significant correlation between physical comorbidity as recorded in the patient's EMR with lack of adherence. These patients may benefit from demonstration of correct eye drop technique with or without the use of adjuncts to help with medication delivery.

Good medication adherence is challenging in chronic conditions, adversely affecting patient health, and in turn, costing the NHS. Defining the extent of the problem and helping to improve adherence is important given 
prevalence of glaucoma not only in the UK, but worldwide. The effect of glaucoma on the NHS is considerable; with around 1 million hospital eye service visits annually. ${ }^{2}$

Glaucoma medication adherence can vary with the type of medication prescribed. Previous studies have shown patient adherence to be better with prostaglandin analogues compared with other medication classes. ${ }^{13}$ Due to our relatively small sample size, it is possible that a true difference in adherence with prostaglandin analogues was not detected in this study.

Chronic diseases such as diabetes have been associated with reduced medication adherence due to problems in lifestyle adjustment in order to incorporate the use of additional medications into their regime. ${ }^{20}$ We did not identify any correlation between presence of such chronic conditions and adherence; however this may be due to our smaller patient numbers.

It can be difficult for ophthalmologists to accurately determine patient adherence with glaucoma treatment. ${ }^{21}$ Self reported estimates of adherence are also unreliable with patients tending to overestimate their adherence rates. ${ }^{13,22}$ A study undertaken by Kass et al ${ }^{21}$ showed the 30-day patient recorded adherence rate in a medication $\log$ to be $99 \%$, significantly higher than the rate of $76 \%$ measured by an eye drop monitor. Furthermore, physicians were unable to accurately identify, which patients had adherence rates of $<50 \%$ compared to $>90 \%$. Our study was conducted using prescriptions issued as a proxy to measure adherence rates with glaucoma treatment. Advantages of this method are the ability to include large numbers of patients and the facility for results to be analysed by drug class. However, this method does not allow for recognition of the reasons for any differences in adherence. While assessing whether all prescriptions issued were filled at pharmacies or medications retrieved would have provided useful information on the validity of our method of measuring adherence, we felt that this method was better than patient self-reporting.

Approaches to improve patient adherence with glaucoma medications outlined by Osterberg and Blaschke include patient education and motivation through highlighting the importance of adherence, providing literature to take away reinforcing information provided at the time of the clinic visit, and reviewing medication regime at each regular, follow-up visit. ${ }^{6}$ In addition, discussions about medication adherence at each clinic visit, especially if the patient is from a group most likely to default with medications or have a tendency for wastage were found to be most useful. Checking and demonstrating eye drop technique and prescribing adherence aids, if necessary, is of paramount importance. Providing easy to read written instructions of treatment intervals and timing and helping patients to integrate medication regimens into their lifestyles is also suggested. ${ }^{23}$

Most GP surgeries currently use EMR, which allows poor and non-adherence (a delay in collecting a prescription from the GP when it would otherwise be due) to be highlighted on the patient's health records when reviewing the patient's prescription record. This information is relatively easy to access when reviewing a patient's health record. Identification of patients within a practice with poor adherence to glaucoma medications is possible through EMR on an individual patient basis, and would require more time to carry out a search to identify all practice patients. However, patients of a particular age group with poor glaucoma medication adherence can be easily identified and targeted with specific educational interventions to improve adherence in order to minimise visual loss. GPs are therefore in a unique position to identify patients with poor adherence to glaucoma medications. Patient education groups and individualised educational interventions in a face-to-face setting or over the telephone have been shown to improve patient understanding of the condition and medication adherence. ${ }^{24}$ A Cochrane review by Waterman et al ${ }^{25}$ showed that there was some evidence that patient education combined with personalised interventions improved adherence and simplified drug regimes could be of benefit, however, there was insufficient evidence to recommend a specific intervention. Limitations to our study consists of not including the length of time patients had been on glaucoma medication to ascertain if there was any correlations between the length of time and adherence, as shown in a previous study by Vanelli et al. ${ }^{26}$

Our study demonstrates that younger patients tended to have the worse adherence of all age groups; it is imperative that this group of patients are targeted for educational interventions as it is important they understand the importance of regular use of medications to prevent visual loss in their expected lifespan. Older patients (>90 years) also need educational intervention to improve their drop instillation technique and to provide help with adherence aids. GPs are well placed to identify such patients to allow interventions targeted at improving adherence to be applied in the primary care setting, potentially minimising unnecessary visits to the hospital eye service. Further research into the clinical impact of non-adherence and interventions to improve glaucoma medication adherence, which can be delivered in a primary care setting is needed. 


\section{Summary}

\section{What was known before}

- Distribution of resources and management of conditions in primary care is becoming increasingly important.

- It can be difficult for ophthalmologists to accurately determine patient adherence with glaucoma treatment.

- Self-reported estimates of adherence are unreliable with patients tending to overestimate their adherence rates.

\section{What this study adds}

- Patients poorly compliant with glaucoma treatments can be identified by the data that are routinely collected by general practices.

- Our results show that younger patients tended to have the worst adherence of all age groups; it is imperative that this group of patients are targeted for educational interventions as it is important to prevent visual loss in their expected lifespan.

- Suggestions include providing educational interventions in the primary care setting by GPs and other healthcare professionals (such as nurses or optometrists) in order to improve adherence.

\section{Conflict of interest}

The authors declare no conflict of interest.

\section{References}

1 Pascolini D, Mariotti SP. Global estimates of visual impairment. Br J Ophthalmol 2012; 96(5): 614-618.

2 National Collaborating Centre for Acute Care (UK). Glaucoma: Diagnosis and Management of Chronic Open Angle Glaucoma and Ocular Hypertension. National Collaborating Centre for Acute Care (UK): London, 2009.

3 Heijl A, Leske MC, Bengtsson B, Hyman L, Bengtsson B, Hussein M. Reduction of intraocular pressure and glaucoma progression: results from the early manifest glaucoma trial. Arch Ophthalmol 2002; 120(10): 1268-1279.

4 Chawla A, McGalliard JN, Batterbury M. Use of eyedrops in glaucoma: how can we help to reduce non-compliance? Acta Ophthalmol Scand 2007; 85(4): 464-464.

5 Tsai JC, McClure CA, Ramos SE, Schlundt DG, Pichert JW. Compliance barriers in glaucoma: a systematic classification. J Glaucoma 2003; 12(5): 393.

6 Osterberg L, Blaschke T. Adherence to medication. N Engl J Med 2005; 353(5): 487-497.

7 Schuman JS. Antiglaucoma medications: a review of safety and tolerability issues related to their use. Clin Ther 2000; 22(2): 167-208.

8 Sleath BL, Krishnadas R, Cho M, Robin AL, Mehta R, Covert D et al. Patient-reported barriers to glaucoma medication access, use, and adherence in southern India. Indian J Ophthalmol 2009; 57(1): 63-68.

9 Nordmann J-P, Denis P, Vigneux M, Trudeau E, Guillemin I, Berdeaux G. Development of the conceptual framework for the Eye-Drop Satisfaction Questionnaire (EDSQ) in glaucoma using a qualitative study. BMC Health Serv Res 2007; 7(1): 124.

10 Owen CG, Carey IM, de Wilde S, Whincup PH, Wormald R, Cook DG. Persistency with medical treatment for glaucoma and ocular hypertension in the United Kingdom: 1994-2005. Eye (Lond) 2009; 23(5): 1098-1110.

11 Dreer LE, Girkin C, Mansberger SL. Determinants of medication adherence to topical glaucoma therapy. J Glaucoma 2012; 21(4): 234-240.

12 Lee MD, Fechtner FR, Fiscella RG, Singh K, Stewart WC. Emerging perspectives on glaucoma: highlights of a roundtable discussion. Am J Ophthalmol 2000; 130: S1-S11.

13 Nordstrom BL, Friedman DS, Mozaffari E, Quigley HA, Walker AM. Persistence and adherence with topical glaucoma therapy. Am J Ophthalmol 2005; 140(4): 598-606.

14 Royal Pharmaceutical Joint Formulary Committee. British National Formulary 65 (edn). BMJ Group and Pharmaceutical Press: London, UK, 2013.

15 Dasgupta S, Oates V, Bookhart BK, Vaziri B, Schwartz GF, Mozaffari E. Population-based persistency rates for topical glaucoma medications measured with pharmacy claims data. Am J Manag Care 2002; 8(10 Suppl): S255-S261.

16 Zhou Z, Althin R, Sforzolini BS, Dhawan R. Persistency and treatment failure in newly diagnosed open angle glaucoma patients in the United Kingdom. Br J Ophthalmol 2004; 88(11): 1391-1394.

17 Olthoff C, Schouten J, Vandeborne B, Webers C. Noncompliance with ocular hypotensive treatment in patients with glaucoma or ocular hypertensionan evidencebased review. Ophthalmology 2005; 112(6): 953-961.e7.

18 Lacey J, Cate H, Broadway DC. Barriers to adherence with glaucoma medications: a qualitative research study. Eye (Lond) 2009; 23(4): 924-932.

19 Sleath B, Robin AL, Covert D, Byrd JE, Tudor G, Svarstad B. Patient-reported behavior and problems in using glaucoma medications. Ophthalmology 2006; 113(3): 431-436.

20 Nagelkerk J, Reick K, Meengs L. Perceived barriers and effective strategies to diabetes self-management. J Adv Nurs 2006; 54(2): 151-158.

21 Kass MA, Gordon M, Meltzer DW. Can ophthalmologists correctly identify patients defaulting from pilocarpine therapy? Am J Ophthalmol 1986; 101(5): 524-530.

22 Kharod BV, Johnson PB, Nesti HA, Rhee DJ. Effect of written instructions on accuracy of self-reporting medication regimen in glaucoma patients. J Glaucoma 2006; 15(3): 244-247.

23 Schwartz GF. Adherence and persistence. Shaarawy TM (ed), Glaucoma Medical Diagnosis and Therapy, Vol 1. Saunders Elsevier: Philadelphia, PA, USA, 2009, pp 489-494.

24 Chen X, Chen Y, Sun X. Notable role of glaucoma club on patients' knowledge of glaucoma. Clin Experiment Ophthalmol 2009; 37(6): 590-594.

25 Waterman H, Evans JR, Gray TA, Henson D, Harper R. Interventions for improving adherence to ocular hypotensive therapy. Cochrane Database Syst Rev 2013; 4: CD006132.

26 Vanelli M, Pedan A, Liu N, Hoar J, Messier D, Kiarsis K. The role of patient inexperience in medication discontinuation: a retrospective analysis of medication nonpersistence in seven chronic illnesses. Clin Ther 2009; 31(11): 2628-2652. 